\title{
Altdeutscher Durst im Spiegel des Auslandes.
}

\author{
Von \\ PAUL FRAUENSTÃDT.
}

Wo immer in auslāndischen Schriften des Mittelalters von den Untugenden der Deutschen die Rede ist, steht deren Trunksucht stets im Vordergrunde. Von allen ausländischen Schriftstellern haben die Italiener am frühesten den unauslöschlichen Durst der Deutschen zur Zielscheibe ihres Spottes gemacht. Mit dem Ausgang des Mittelalters hatte die alte Unsitte der Deutschen immer stārker zugenommen, und im 16. Jahrhundert stieg bekanntlich die Trunksucht aller Stānde ins Ungemessene. ${ }^{1)}$ Seit dem 15. Jahrhundert vermehren sich denn auch die Stimmen, die über die Schwäche der Deutschen sich vernehmen lassen. Hören wir zunāchst die Italiener.

Der italienische Humanist Gian-Francesco Poggio (1380 bis 1459), der als päpstlicher Sekretär sich zu wiederholten Malen in Deutschland aufgehalten und in dortigen Klöstern auf alte Handschriften gefahndet hat, hāngt in einem Briefe an den Kardinal Cesarini den Deutschen die Schmähworte an: „Ehemals waren die Deutschen eine kriegerische Nation; jetzt suchen sie statt in Waffen- ihre Mannhaftigkeit nur noch in Weingefechten; ter gröBte Held ist, wer am meisten vertragen kann, “') und schiebt die Schuld an der schmāhlichen Flucht vor den Hussiten bei

1) Vgl. dazu Steinhausen, Geschichte der deutschen Kultur. S. 507 f. Siehe ferner die Belege weiter unten.

2) Fuit aliquando bellicosa germanica natio, nunc pro armis vino pugnant et crapula: tantumque habent virium, quantum vini capere possunt. 
TauB scherzend darauf, daß der Kardinal seinen Truppen nicht genügend Wein gespendet und gehofft habe, mit nüchternen Deutschen zu siegen. ${ }^{1}$ ) An einer anderen Stelle (.Invectiva in Felicem antipapam ${ }^{a}$ ) nennt er die Deutschen "niemals nūchterne Geschöpfea. Wenn sie von Wein und Schmausen überwāltigt dalägen, vermöge man nicht zu unterscheiden, ob sie tot oder lebendig seien. $\left.{ }^{9}\right)$ Nicht glimpflicher äuBert sich über die deutsche Trunksucht der pāpstliche Legat Campano, der 1471 zum Regensburger Reichstage geschickt wurde, um durch die Gewalt seiner Rede die Deutschen zum Türkenkriege anzufeuern, in dem Spottverse:

Arce sedet Bacchus, cessit neglectus Apollo:

Nil hic est aliud vivere quam bibere.

Italienischer Quelle entstammt anscheinend auch das ähnlich lautende Distichon:

Perdidit haud temere priscas Germania laudes,

Cui nihil est aliud vivere quam bibere.

Wie die Italiener hat sich auch der englische Dichter John Owen in verschiedenen Epigrammen über die Trunksucht der Deutschen lustig gemacht, von denen besonders das folgende:

Si latet in vino verum, ut proverbia dicant, Invenit verum Teuto vel inveniet,

sich durch zierlichen Witz auszeichnet. Auch Shakespeare hechelt im "Kaufmann von Venedig" (I, 2) die sprichwörtliche Trunksucht der Deutschen durch. Auf die Frage Nerissas, wie ihr der junge Deutsche, des Herzogs von Sachsen Neffe, gefalle, läBt er die Porzia antworten: "Sehr abscheulich des Morgens, wenn er nüchtern ist, und höchst abscheulich des Nachmittags, wenn er betrunken ist.... Aus Furcht vor dem Schlimmsten bitte ich dich, setze einen Römer voll Rheinwein auf das falsche Kāstchen: denn wenn der Teufel darin steckt und diese Versuchung ist von außen daran, so weiß ich, er wird es wählen." Ein franzōsischer Edelmann, Alexandre de Pontaymerie Seigneur de Focherau, nannte

1) Voigt, Wiederbelebung des klassischen Altertums. II, 312.

2) Atqui homines, dii boni, somnolenti, stupidi, stertentes, nunquam sobrii, hominibus odibiles et deo, qui vivi an mortui essent, vino atque epulis obruti, non discerneres. 
Deutschland ein Reich der Trunkenbolde, und noch viel schärfer und drastischer sprach sich ein anderer Franzose, der berühmte Henricus Stephanus (Henri Etienne), aus.

Niemand ärgerte sich über diese Sticheleien mehr als der geschätzte Geograph Martin Zeiller, der, wenn er auch den Nationalfehler der Deutschen nicht leugnet, in seinem "Itinerarium Germaniae" ("Teutsches Reisebuch ") den anderen Nationen gleichfalls Neigung zum Trunk vorwirft. Vor allen andern, meinte er, hătte doch Owen seinen Mund halten sollen, der selber keinem guten Trunk aus dem Wege gegangen und in London weit ofter in den Tavernen und Weinhāusern als in seinen vier Pfählen zu finden gewesen sei. Was die Englānder betrifft, so mochte Zeiller recht haben. Auch sie waren stramme Zecher. Von wo hätte Shakespeare, der niemals aus England herausgekommen ist, das Vorbild zu seinem Sir John Falstaff entnommen, wenn nicht aus englischen Wein- und Bierhäusern? Bei Italienern, Spaniern und Franzosen schwankte man sogar, ob die Engländer oder die Deutschen die mãchtigsten Trinker seien. So lautet ein altfranzösisches Sprichwort:

Les plus belles femmes sont en Flandre,
Les plus beaux hommes en Allemagne,
Les plus grands en Dannemarc,
Les meilleurs buveurs en Angleterre.

Auch der englische Reisende Moryson bestätigt in seinem "Itinerary “ (London 1617), daB in langen und tiefen Trünken seine Landsleute den Deutschen überlegen seien, wogegen diese "eine Ewigkeit" am Zechtische zubrächten.

Moryson ist für die Kenntnis der deutschen Lebensgewohnheiten kurz vor Ausgang des 16. Jahrhunderts eine noch viel zu wenig beachtete Quelle. Lebensgetreue Schilderung von Land und Leuten gehörten nicht zu den Gepflogenheiten der deutschen Schriftsteller des Zeitalters. Es mangelte ihnen dazu noch mehr an Sinn als an Begabung. In ihren geographischen Werken und Reisehandbūchern überwiegt das historisch-antiquarische Interesse. Selbst die flotter geschriebenen Reisetagebücher des Samuel Kiechel und Philipp Hainhofer stehen trotz ihres tieferen Eindringens in die Lebensgewohnheiten der von ihnen besuchten Länder und 
Gegenden an Fülle wie an Schärfe der Beobachtungen hinter Morysons Reisewerken beträchtlich zurück. Moryson, gleich hervorragend durch gelehrte wie durch Weltbildung, hat sich zwei Jahre (1591 und 1592) zur Erlernung der Sprache in Deutschland aufgehalten und das Land in allen Teilen kennen gelernt, indem er, der damaligen Touristensitte entsprechend, die Frūhjahrs- und Herbstzeit zu ausgedehnteren Touren benutzte, während er die Winter und Sommer auf den Universităten Wittenberg, Leipzig und Heidelberg zubrachte. Seinen wiederholt ausgesprochenen Grundsatz an sich selber betătigend, daß man, um Land und Leute genügend kennen zu lernen, mit dem Ausländer in Ausländers Weise verkehren müsse, bewegte er sich, dieselben in ihrem täglichen Tun und Treiben scharf beobachtend, viel in deutschen bürgerlichen Kreisen. Dies und seine Geistesrichtung, die dem Kleinen und Alltäglichen dasselbe Interesse entgegenbrachte wie dem GroBen und AuBergewöhnlichen, machen, daB man in seinem Reisewerke über die damaligen Lebensgewohnheiten der breiten Volksmassen mehr erfährt als aus den einheimischen Schriftstellern. Für die Glaubwürdigkeit seiner Berichte, auch da, wo er mißbilligt und tadelt, bürgt die Unparteilichkeit, mit der an vielen Stellen seines Werkes die guten Seiten des deutschen Volkscharakters ohne Vorbehalte und Einschränkungen von ihm anerkannt werden, eine Eigenschaft, die ihn von den gebildeten Elementen unter den Franzosen und Italienern seiner Zeit vorteilhaft unterscheidet. Er blickt nicht mit dem Hochmut der italienischen Humanisten auf das deutsche Volk und dessen manchmal plumpe Umgangsformen herab, und wenn er angreift, geschieht es niemals in der sarkastischen Weise des Erasmus und der Franzosen.

Moryson beginnt unter der Überschrift "The Germans diet" (III, 81 ff.) seinen Bericht über die Lebenshaltung der Deutschen in Speise und Trank mit der charakteristischen Bemerkung: "Die Lebensweise der Deutschen ist einfach und bescheiden, wofern man von ihrer Unmäßigkeit im Trinken absieht. Da sie sowohl in der Kleidung wie im Zuschnitt des häuslichen Lebens eher zur Einschränkung als zu Prunk und Aufwand neigen, sind sie mit einem Stück Fleisch und Brot zufrieden, wenn 
sie nur hinlānglich zu trinken haben und keine Not an Holz leiden, ihre Stube zu heizen. " ") Um einen Beweis von der Einfachheit der Bewirtung selbst bei festlichen Gelegenheiten zu geben, beschreibt er das Hochzeitsmahl im Hause eines angesehenen Leipziger Ratsherrn, zu dem er sich eine Einladung verschafft hatte. Die Bewirtung war allerdings selbst nach damaligen Verhältnissen, vielleicht unter dem Einflusse der luxusbeschränkenden Hochzeitsordnungen, nichts weniger als glänzend. Sie bestand im wesentlichen nur aus Rinds- und Hammelbraten, Fisch, Kuchen und Käse. Weder Wild noch Geflügel noch ein Nachtisch von SüBigkeiten kam zur Verwunderung des Englānders auf die Tafel. Um so reichlicher floB der Wein. Ganze Fãsser wurden in das Zimmer geschrotet und neben den Gästen auf einen Tisch gesetzt, und so unmäBig wurde dem Wein zugesprochen, daß schon zwei Stunden nach Beginn des Essens allgemeine Trunkenheit unter den mānnlichen Gāsten herrschte und keiner sich am andern Morgen entsinnen konnte, was er hinterher getan oder gesprochen hatte.

Moryson bezeichnet es als eine Eigentümlichkeit der Deutschen, daB sie aus Lust am Essen die Hauptmahlzeit des Tages ungebührlich ausdehnen. "Sie sitzen," so berichtet er, "lange bei Tisch, und selbst auf Reisen erheben sie sich nicht eher vom Mittag- und Abendbrot, bis sie, wenn auch langsam, alles aufgegessen haben, was vor ihnen steht. Und sie können nicht verächtlicher von einem Wirte sprechen, als wenn sie sagen: „Ich hab' mich da nicht satt gefressen.“ Moryson verdanken wir zugleich eine genauere Kenntnis der deutschen Gasthauskūche, von der ein Gemisch aus Sauerkraut, Würsten, Hühnern und allerlei gebratenem Fleisch dem Engländer besonders gemundet zu haben scheint. Auch in vielen andern Stücken fand die deutsche Küche des Engländers Beifall; ${ }^{2}$ ) [hōchlich miBfiel ihm dagegen das starke Salzen

1) Vermutlich hatte Moryson hierbei die Vorliebe der Deutschen für heiße Stuben im Sinn, über die sich schon Erasmus von Rotterdam in seiner drastischen Schilderung der deutschen Wirtsstuben beklagt.

2) So rühmt er die Schmackhaftigkeit der langen Bratwürste, die Zubereitung der Fische mit Reis, des Backobstes mit Zimt und Butter und des Kalbskopfes, der im nōrdlichen Deutschland unzeriegt auf den 
der Speisen, das, wie er sagt, den Zweck hatte, Durst zu erzeugen. ${ }^{-1}$ ) Darum durften auch, wie er weiter mitteilt, bei keiner Gasterei Śchinken, gepökeltes Rindfleisch und andere scharf gesalzene Speisen fehlen, und hiermit nicht genug, wurden nach Entfernung des Tischtuches Bohnen, welsche Nüsse und mit Salz und Pfeffer bestreute Brotschnitten aufgesetzt, von denen schon ein einziger Bissen zum Trinken reizte.

Moryson kommt auf die UnmäBigkeit der Deutschen im Trinken, von der er, wiewohl nicht ganz mit Recht, nur die Frauen ausnimmt,") zu wiederholten Malen mit einer Ausführlichkeit zu sprechen, die nur dadurch erklärlich wird, daB sich diese Erscheinung dem Auge des Ausländers auf Schritt und Tritt in einer von ihm sonst noch nirgends wahrgenommenen Massenhaftigkeit und Methodik aufdrăngte. Lassen wir ihn darüber selber reden. „Die Deutschen,“ so beginnt er seine Ausführungen, "sind, wenn man die Wahrheit sagen soll, in hohem Grade dem Trunke ergeben, während man im übrigen kaum von einem sie

Tisch kam. Beim ersten Male, so erzāhlt er, hätte ihm und seinen Landsleuten der Anblick des zāhnefletschenden Ungetüms einen Schrecken eingejagt, indessen könne er sich nicht erinnern, jemals etwas seinem Geschmack Zusagenderes gegessen zu haben.

1) Als Moryson sich einmal bei seinem Wirt über das starke Salzen beklagte, weil es seinem Körper nicht zusagte, erwiderte ihm dieser halb im Ernst, halb scherzweise, daB der Gebrauch des Salzes in der Bibel anbefohlen sei, indem er dabei den Spruch anfūhrte: "Lasset eure Rede mit Salz gewürzet sein."

2) Er rühmt ihre große Mäßigkeit im Essen und Trinken. Es bestände bei ihnen die Sitte, ihren Männern oder Freunden Bescheid zu tun, indem sie am Becher nippten, niemals aber habe er ein keusches Weib (und das seien sie fast alle) viel trinken, noch weniger betrunken gesehen. Auch der bereits genannte Franzose de Pontaymerie, welcher Deutschland ein Reich der Trunkenbolde und Vollsäufer nannte, rühmt den deutschen Frauen nach, sie seien so nüchtern und mäBig, daß man auch nicht eine einzige fände, die dem Trunke ergeben sei, ja, die auch nur einmal zu viel getrunken hätte. Von den Frauen der Mittelstānde mag das mit Recht geiten, weniger von der Volkshefe und von den Hofdamen. Heinrich IV. von Frankreich wollte keine deutsche Prinzessin zur Frau, indem er sagte: "Je croirais toujours avoir un pot de vin auprès de moj “, und bekannt ist die Trunksucht der Prinzessin Anna v. Sachsen, Gemahlin des großen Wilhelm von Oranien. 
beherrschenden Nationalfehler hört. Wie die Nation im allgemeinen und jedes einzelne ihrer Glieder, werden auch ihre Künstler und Gelehrten, obwohl sie so tun, als lebten sie für nichts als ihre Kunst und Wissenschaft, infolge der stetigen Übung im Trinken sehr bald funbesiegbare Meister in dieser Fertigkeit. Wenn die Stadttore geschlossen werden, und die Leute, die in den Vorstādten wohnen, hinausgehen, taumeln sie von einer Seite zur andern, stolpern, fallen in den Kot und spreizen die Beine, als sollte zwischen diesen ein Wagen durchfahren. Kommen sie dann wieder auf die Füße, so rennen sie an jeden Pfosten, Pfeiler und des Weges Kommenden an. Selbst die Stadttore scheinen für sie nicht weit genug, ausgenommen, die Mauern würden niedergerissen.

Spectatum admissi risum teneatis amici.

Die reicheren Leute suchen zwar ihre UnmäBigkeit meistens zu verheimlichen, indem sie sich zu Hause halten, dagegen gibt der gemeine Haufe tăglich ein solches Schauspiel. Ich weiß nicht, was den Deutschen die Gesellschaft von Trunkenbolden so anziehend macht, da niemand sich durch andere Eigenschaften so viel Freunde machen kann als gerade damit, so daB, wenn jemand gern gesehen sein will oder ihre Sprache zu erlernen wünscht, er sich bis zu einem gewissen Grade im Trinken üben muB. Wenn sie beim Trunk sitzen und es kommt jemand ins Zimmer, sei es auch ein Fremder oder ein Ausländer, so beschwören sie ihn bei dem Bande der Freundschaft, bei seines Vaters Adel, bei seiner Mutter Keuschheit, innen Bescheid zu tun, und wenn das nicht hilft, nōtigen sie ihn dazu, indem sie ihm zurufen: ,Kannst du nicht saufen und fressen, so kannst du keinem Herrn wohl dienen.' Jeder am Tische begrüBt ihn mit einem Becher, die er alle bis auf die Neige leeren muB, bevor er zu ihrer Gesellschaft zugelassen wird, so daB einem besser ist, unter seine Feinde mit Fechten als unter seine Freunde mit Trinken zu geraten. Sie sind am Zechtische selten sehr lustig und redselig, sondern rufen nur zuweilen einander zu: ,Seid fröhlich, trinkt aus!, und wie jeder Psalm mit einem Gloria, so endet jedes ihrer Gespräche mit einem: ,Ich bring's euch, ich trinke euch zu.' Aus Scherz kneipen sie ihren nächsten Nachbar - und zwar ganz gehörig - in den 
Arm oder ins Bein, und das geht so weiter in der Runde herum. Auch den Humpen lassen sie herumgehen und sagen, diese Sitte habe ihren Ursprung in einer drolligen oder vielmehr niederträchtigen Handlung eines unehrerbietigen Sohnes, der eine Ohrfeige von seinem Vater erhalten hatte, und da er nicht wagte, den Schlag zu erwidern, gab er seinem Nachbar eine ebenso derbe Maulschelle, wie er selber bekommen, und forderte diesen scherzweise auf, sie weitergehen zu lassen, indem er sprach: ,LaBt's umgehen, so kriegt der Vater auch was.' Wer beim Kreisen des Humpens den letzten Rest erwischt, dem prophezeien sie mit Hohngelāchter, da $B$ er eine alte Vettel zur Frau bekommen werde. In Sachsen trinken immer zwei aus einem Glase, und wenn einer es erhält oder seinem Mittrinker reicht, geben sie genau Obacht auf gewisse Stiftchen oder andere Merkzeichen, die inwendig angebracht sind, damit von dem Getrānk der eine genau so viel bekommt wie der andere (that they may divide the drink by the equal balance of Justice). Manchmal nehmen sie drei Gläser auf einmal, setzen jedes auf einen Finger und trinken sie zu gleicher Zeit aus. Sie nennen das , die Krönung des Kaisers'. Sind sie recht lustig, so lassen sie ein Bierspiel los, das "Kurlemurlebuff heiBt. Es besteht in der Berührung des Glases, des Bartes, des Tisches, Pfiffen und Schnippen mit den Fingern nach bestimmten Regeln in einer so raschen, seltsamen Aufeinanderfolge, daB es eine Herkulesarbeit ist, den Bewegungen zu folgen. Wer den geringsten Fehler macht, muB zur Strafe einen vollen Humpen spenden. “ ')

Dem Ausländer machte sich die deutsche Trinklust nicht nur in mancherlei Belästigungen durch betrunkene Mitreisende,

1) Wie zuverlässig und wahrheitsgetreu Moryson in seinen Mitteilungen ist, ergibt sich aus der Sammlung der Trinkregeln, die zuerst im Jahre 1616, also gerade 25 Jahre nach Morysons Aufenthalt in Deutschland, unter dem Titel "Jus potandi oder Zechrecht“ im Druck erschien. Die meisten der oben geschilderten Zeremonien und Gebräuche werden darin erwähnt, insbesondere das "Curl-Murl-Puff“, von dem es daselbst heiBt: "Da dann der Bart bald da bald dort gewischet, bald da und dort itzt mit den FüBen getappet, bald mit den Fingern geschnipfft, eins gepfiffen und sonsten viel seltsame phantastische Possen gebrauchet werden." 
sondern mehr noch durch die erhebliche Verteuerung des Reisens in unangenehmer Weise fühlbar. Der deutsche Gastwirt war zu bequem, jedem Reisenden die Zeche besonders aufzustellen; er rechnete zusammen, was von den Gāsten an Speisen und Getränken insgesamt verzehrt worden war, und dividierte den Betrag mit der Zahl der Gāste, so daß, mochte einer viel oder wenig verzehrt haben, er so viel bezahlen muBte wie der andere. Schon Erasmus von Rotterdam (1467-1536) beschwert sich in den "Colloquien" über diese Unbilligkeit, bei welcher der starke Trinker āußerst vorteilhaft, der schwache um so schlechter abschnitt; denn es gab Gäste, die das Doppelte von dem, was die Mahlzeit kostete, in Wein vertilgten, trotzdem aber nicht einen Heller mehr bezahlten als jeder andere. Bei den Deutschen muB wohl im allgemeinen dieser Wirtshausbrauch viel Anklang gefunden haben, denn er bestand mit einer unwesentlichen Änderung auch noch zu Morysons Zeit. Der Wirt warf zwar nicht mehr die ganze Zeche in einen Topf, aber doch das ganze Getrānk, das nach dem Abendbrot vertilgt wurde. Wer sich, sobald das Tischtuch entfernt war, nicht sofort zu Bett begab, sondern an dem sogenannten "Schlaftrunk" beteiligte, mußte, wenn er auch nur sehr wenig trank, für sein kleines Quantum ganz dasselbe bezahlen wie die, welche bis zum Morgen zechten. Da alle Deutschen stramme Trinker waren, machte dieser Gebrauch, wie Moryson sagt, das Reisen in Deutschland für die Fremden erheblich teurer als in andern Ländern.

Luther hat in der Auslegung des 101. Psalms den Ausspruch getan: "Es muB aber ein jeglich Land seinen eigenen Teufel haben, Welschland seinen, Frankreich seinen. Unser teutscher Teufel wird ein guter Weinschlauch sein und muß Sauff heißen, $\mathrm{da}$ er so durstig ist, daB er mit so groBem sauffen weins und biers nicht kan gekület werden. Und wird solcher ewiger Durst teutschen Landes Plage bleiben (hab' ich Sorg) bis an den jüngsten Tag." Seitdem ist viel in Prosa wie in Versen gegen den "Saufteufel " und das bis zum gröBten Exzeß ausgeartete Zutrinken geschrieben worden. Nicht nur aus Humpen, Kannen, Krügen, sondern, wie in einer dieser Schriften, dem "Sendbrief an die vollen Brüder" (1565), zu lesen ist, auch aus Schüsseln, Töpfen, 
Kāsenāpfen, Waschbecken, Handfāssern, Fischpfannen, ja sogar aus Hüten und Schuhen trank man einander zu. Selbst in die Frauen und Kinder war der Saufteufel gefahren. "Es üben," heißt es in derselben Schrift, ${ }^{n}$ solches Laster jetzund nicht allein die Mannspersonen, sondern auch die Weiber, nicht allein die Alten, sondern auch die jungen Kinder, die kōnnen allbereit einander ein halbes zutrinken. Die Eltern lehrens auch wohl ihre Kinder. Nun, laß sehen, spricht der Vater zum Söhnlein, was du kannst. Bring ihm ein halbes oder ganzes." Und gar beweglich singt der Dichter von "Ein Lied wider das vollsauffen und Trunkenheit, getruckt zu Frankfurt am Main 1565":

Die Weibesleut auch heben an,

Einander zuzutrinken, Volle und halbe wie die Mann, Mein hertz will mir entsinken, Wenn ich bedenk die sūnde schwer Und allen schaden, so folgt her Aus ÜberfluB des Trinkens.

So sehr hatte in der Tat die Trinksucht wie ein bōser Dämon des ganzen Volkes ohne Unterschied des Standes, Geschlechts und Alters sich bemāchtigt, daB schon auf den Reichstagen in Worms, Lindau und Freiburg (1495-1498) über MaBregeln gegen das Zutrinken von "gleichen, vollen und halben " beraten wurde, worin gerade die Fürstenhōfe und der Adel das übelste Beispiel gaben, so daB der Reichstag von 1512 mit Strafen einzuschreiten beschloB. Wie wenig Erfolg aber diese in den Reichspolizeiordnungen erneuerten Verbote hatten, ${ }^{1}$ ) beweisen $\boldsymbol{u}$. a. Morysons Ausführungen, indem der Englānder, an jene Reichserlasse anknūpfend, nicht ohne einen Anflug von Ironie und sittlicher Entrüstung in die Worte ausbricht: "Aber zeigt mir einen Fürsten, der diesem Laster nicht fröhnt und seine Hofleute deshalb strafen dürfte. Sah ich doch mit eigenen Augen einen Herzog bei der Beerdigungsfeier für einen seiner nächsten fürstlichen Vettern so hartnāckig trinken, um seinen Gram zu lindern, daB alle seine Sinne und Geister benebelt waren, und von vielen anderen anwesenden Fürsten (Ver-

1) Die Herren vom Adel tranken einander mit dem Spottrufe zu: -Es gilt dem Reichsabschied." 
zeihung, daB ich die Wahrheit spreche) sah ich auch nicht einen einzigen nūchtern. Wie würden diese Fürsten erst bei einer Hochzeit gebügelt haben! Zeigt mir einen Ratsherrn unter so vielen Tausenden, der im Gefühle eigener Unschuld wagen dürfte, die anderen zu strafen. Zeigt mir - ich schäme mich, es $z u$ sagen, aber wahr bleibt wahr - zeigt mir, sage ich, einen Diener von Gottes Wort, der gegen die Ausschweifung im Trinken predigen würde."

Ganz so schlimm stand es nun zwar um die Geistlichkeit nicht, denn immer gab es einzelne Geistliche, die nicht müde wurden, gegen den Hexensabbat des Trinkens und Zutrinkens als den "Verderber", wie der Reichsabschied von 1512 sagt, "von Ehre, Seele, Vernunft, Leib und Gut “ ihre Stimme zu erheben. Nichtsdestoweniger stand es noch immer schlimm genug, wenn man z. B. liest, daB in Hessen ein ziemlich groBer Teil der Geistlichen mit den Bauern in den Dorfschänken zechte und sich prügelte, da $B$ die Bauern die Bierkrüge mit in die Kirche nahmen, sich wãhrend der Predigt daraus zutranken und die Deckel dem Pfarrer klappernd ins Wort fallen lieBen, ja, daB an hohen Festtagen ganze Fässer voll Wein und Bier in die Kirche geschrotet und dort ausgetrunken wurden. Auf den Universităten trank nicht bloB der Student, es trank auch der Professor; häufig tranken beide in brüderlicher Vereinigung, weil viele Professoren, die Theologen nicht ausgenommen, zur Verbesserung ihres Einkommens neben ihrem Lehramt den Bier- und Weinschank betrieben. Herzog Julius von Braunschweig verwarnte 1597 die Helmstedter Universităt, ihm keine "versoffenen " Professoren in Vorschlag zu bringen. Und als der gelehrte Landgraf Moritz von Hessen, „obwohl für seine eigene Person ein MäBigkeitsexempel unter den damaligen Fürsten," der Universität Marburg 1615 seinen etwas trunkfälligen Privatsekretär Thys zu einer Professur empfahl und die Universitāt denselben ablehnte, schrieb ihr der Fürst ironisch zurūck: „es sei ihm ihre abschlägige Antwort den Thysium betreffend unvermuthend gewesen; und ob er (Thysius) wohl hiebevor ex corruptelis aulae aliquid morbi contrahiret haben möge, so habe er sich doch zu allem unsträflichen Wandel und Wesen erboten... Sollte es auch allein auf unnōtigen Trunk gemeint 
sein, tragen Wir Sorge, daB er zu Marburg viel Brüder finden würde, die mit ihm eure Uns fast unvermuthete repulsam zum Thore hinaustragen müBten, denn Uns leider zu wohl bekannt ist, daB fast in allen Fakultäten gute Zechbrüder und Lucubranten mit unterlaufen. ${ }^{\text {1) }}$ )

Auch mit dem, was Moryson über die Trunkenheit der Ratsherren sagt, hat es seine Richtigkeit. Es macht einen nicht wenig seltsamen Eindruck, daB z. B. der Rat von Frankfurt a. M. sich veranlaBt fand, gegen seine eignen Mitglieder mit Strafandrohungen für Trunkenheitsfälle einzuschreiten. ${ }^{2}$ ) Wie niedrig im Preise ferner in den Adelskreisen die Nüchternheit stand, erhellt aus dem Behagen, mit dem der biedere Hans v. Schweinichen von den schier zahllosen "guten Räuschen" erzählt, die er sich mit seinen Standesgenossen zu Hause und als Reisemarschall des Herzogs Heinrich von Liegnitz auf dessen Bettelreisen durch Deutschland angetrunken hatte. Dieser Herzog Heinrich war selber einer der schlimmsten Trunkenbolde, wie wir aus Schweinichens Denkwürdigkeiten und aus der Lebensbeschreibung des Stralsunder Bürgermeisters Bartholomäus Sastrow wissen, und ein großer Teil seiner regierenden und fürstlichen Vettern gab ihm darin nicht viel nach. Nicht ohne lebhafte Besorgnis lieBen deshalb manche fürstliche Eltern ihre Söhne an fremde Höfe ziehen. "Habe nur Sorge," schreibt die Kurfürstin Friedrich von der Pfalz, als sich ihr Sohn Casimir am Hofe des Markgrafen von Ansbach und Bayreuth aufhielt, "der Markgraf werd' mir ihn krank saufen." Dieselbe Befürchtung spricht aus einem Schreiben des Kurfürsten, als er seinen Sohn Ludwig nach Neuburg zu einer Kindtaufe hatte reisen lassen, weil seine Vettern, Herzog Albrecht zu Bayern und Herzog Christoph zu Württemberg, beides gefürchtete Trinker, ebenfalls dort waren. Und als des Herzogs Julius von Braunschweig Sōhne 1584 den kursiichsischen Hof be-

1) Tholuck, Das akademische Leben im 17. Jahrhundert. I, 44, 41.

2) "Wird ein Trunkener auf der Straße gesehen, so soll jeder Diener des Rathes ermächtigt sein, ihn zu greifen und ins Gefängnis zu führen; und wo die Person aus dem Rathe wäre, soll sie mit doppelter Pöne verfallen sein." Kriegk, Deutsches Bürgertum im Mittelalter. I, 323. 
suchten, bat der Vater in einem Schreiben den Kurfürsten August, dieser "möge sich die jungen Prinzen zu allem Besten văterlich befohlen haben und sonderlich dafür sein, daB sie mit übermäBigem Trinken, welches denselben gar nicht dienlich, unbeschwert bleiben mōchten. " 1) So erzählt auch Graf Froben Christoph von Zimmern (1519-1567), der in der berühmten, nach ihm benannten Zimmerischen Chronik vielfach über das zu seiner Zeit nso entsetzlich “ überhandnehmende Laster der Trunkenheit klagt, von einem Schreiben, in welchem Graf Günther von Schwarzenberg einem Grafen Christoph von Tengern mitteilte, daB auf dem Fürstentage in Naumburg der Rheingraf Philipp Franz mit lauter Malvasier tot getrunken worden sei. Dem Grafen Christoph, erzählt der Chronist, verursachte diese Nachricht groBes Herzeleid, aber nicht von des ehrenwerten Grafen Absterbens wegen, sondern weil er sich bei der wackeren Kompanei nicht auch gegenwärtig befunden und seinesteils dazu geholfen, den Rheingrafen tot zu trinken. "Als ob er vermeinte," setzt der Chronist hinzu, "eine gute, ehrliche That versāumt zu haben." Wie hăufig solche Großtaten der Völlerei in adligen wie bürgerlichen Trinkerkreisen vorgekommen sein mögen und wie man sich ihrer rühmte, ergibt sich aus einer Stelle in dem bereits erwähnten "Sendbrief an die vollen Brüder", in dem es heiBt: "Über das alles hat man solch' Lasters des Saufens und der Trunkenheit kein Hehl, sondern man kitzelt sich damit, als hătte man gar wohl gehandelt. Ja, rühmens auch herrlich, und sagt einer zu dem andern: ,Ich wollte, daB Du năchtens bei uns gewesen wärst, wir waren recht fröhlich. Da ließen wir das Rādlein heruntergehen, es durfte keiner nüchtern daraufkommen. Ich soff sie endlich alle nieder. Der fiel auf die Bank, jener fiel gar hinunter und blieb liegen. Da solltest Du Wunder gesehen haben.' ,Nun reut's mich, spricht der andere, daB ich nicht dabei gewesen, ich wölt Dir einen treuen Gehülfen abgegeben haben.' " "Das Bier," sagt Sebastian Münster in der "Kosmographie“ (3. Buch, c. 439: „Von der Sachsen Sitten und Fruchtbarkeit ihres Landes"), "trinken sie also unmäBig, ja reitzen und zwingen einander $z u$ einem solchen ÜberfluB, daB einemı

1) Zeitschrift für deutsche Kulturgeschichte. N. F. IV, 227. 
Ochsen zu viel wãre. ${ }^{1}$ ) Und das treiben sie den gantzen Tag und auch oft die gantze Nacht, und welcher die andern mit trinken überwindt, der wird darum gelobt und ist ihm eine Ehre, er überkomt auch dadurch ein Kleinod, umb welches er mit trinken zu stritten hat, und zum Zeugnis seiner erjagten Ehre wird er gekrönt mit Rosen oder andern wohlriechenden Krāutern."

Was die Niederdeutschen in Bier, leisteten die Rheinlānder in Wein. Von der MaBlosigkeit, mit der besonders in guten Weinjahren dem Wein zugesprochen wurde, kann man sich ungefāhr eine Vorstellung machen, wenn der Kölner Lizentiat Hermann von Weinsberg in seinem "Gedenkbuch “') erzählt: Im Jahre 1540, als der Wein wegen des warmen Sommers und Herbstes besonders gut geraten war, hätte sich das Volk dergestalt betrunken, daB viele auf der StraBe und an den Hecken "wie die Schweine“ dagelegen hätten. Dieser gute Wein, so fährt er fort, war auch Ursache, daB wir Studenten viel in Gesellschaft zusammenkamen und dermaßen tranken, daß einer nach dem andern umfiel. Auch von dem gesundheits-, ja geradezu lebensgefährlichen ") Zutrinken und Bescheidtun erzāhlt Weinsberg einige Stückchen, so u. a. von zwei Studiengenossen, die sich eines Tages mit ganzen Kannen zugetrunken und dabei dergestalt zugedeckt hatten, daB der eine auf dem Nachhausewege in die Gosse fiel und sich "darin wälzte wie ein Schwein". "Das Trinken wurde leider so stark geübt," fügt Weinsberg hinzu. Wenn der Deutsche von seinen eigenen

1) Moryson erklārt dieses Bier als so dick und unschmackhaft, manchmal wie Medizin, daB ein Fremder glauben würde, es eigne sich eher für die Senkgrube als zum Trinken. Ein Tropfen, der ihm einmal auf die Hand fiel, kam ihm vor wie faules Pfützenwasser.

2) Zeitschrift für deutsche Kulturgeschichte. N. F. I, 566.

3) "Wer weiß nicht," sagt der witzige pseudonyme Verfasser des originellen Jus Potandi, der trotz der Schellenkappe, die er sich aufsetzt, gelegentlich ein Wort der MiBbilligung gegen das wüste Treiben der Saufbrüder nicht unterdrückt, wer weiß nicht, wie viele gute, gesunde Leute, indem sie mit solchem Gesundheittrinken einem andern die seine haben wollen erhalten oder verbessern helfen, sich ihrer eigenen, ja ihres Leibes und Lebens, dessen sie sonst Alters halber noch wohl eine Zeitlang hätten in anderm Wege genießen können, gar elend und muthwillig spoliiret und beraubt haben.“ 
Landsleuten in solchen Ausdrücken sprach, und Weinsberg war darin bei weitem nicht der einzige, wird man es den mäBigeren Italienern und Franzosen so sehr nicht verübeln dürfen, wenn sie den Deutschen Ehrentitel wie: Porco tedesco inebriato, Aleman yvrongne anhingen.

Das Ausland ist zwar schon damals nicht immer gerecht gegen die Deutschen gewesen, aber gerade inbetreff ihrer Trunksucht kann man ihm Ungerechtigkeit des Urteils nicht zum Vorwurf machen. Es war ja nicht allein der häufige Anblick sinnlos Betrunkener, was abstoBend auf die Fremden wirkte, sondern auch die Unflāterei und die Verrohung des gesellschaftlichen Umgangstones, die infolge des wūsten Treibens in bedenklicher Weise um sich gegriffen hatten und von den Gesitteteren im eigenen Lande mit nicht geringerem Ekel empfunden wurden als von den Fremden. Wer wissen will, wie es in dieser Beziehung zuging, braucht ja nur einmal Dedekinds treu nach dem Leben gezeichneten "Grobianus" (verdeutscht durch Caspar Scheidt), das "Jus Potandi“, besonders die Kapitel 7, 12, 37, 43-48, und Mohls geschichtliche Nachweisungen über Sitten und Betragen der Tübinger Studenten des 16. Jahrhunderts nachzulesen. 